\title{
Technical enablers of human errors and violations in construction
}

\author{
Fidelis $\mathrm{Emuze}^{1^{*}}$, and Lesiba $\mathrm{M}$ ollo ${ }^{1}$ \\ ${ }^{1}$ Department of B uilt Environment, Central University of Technology, B loemfontein, Republic \\ of South A frica
}

\begin{abstract}
The performance of public sector projects in developing countries such as South A frica is an on-going concern in a region where injuries and fatalities are almost a daily occurrence in the industry. This reported study assessed the human errors and violations consistently encountered on public sector projects. The feedback from the survey research reinforced the notion that technical matters such as poor planning or the lack of it significantly erode safety system capacities on construction sites. The technical problems are aided by latent conditions such as design errors and negligence. The issues highlighted imply that public sector projects tend to fail to achieve the required conditions of satisfaction by clients. In other words, the reduction of human errors and violations as safety concerns should look beyond the set-up of construction site operations and people in the front line of construction.
\end{abstract}

Keywords: Accident, Construction, Human error, Human violations

\section{Introduction}

There are numerous challenges that construction organisations are struggling to eliminate. One of such problems is the high rate of accidents and incidents related to human errors and violations [1]. This study sought responses to a question that asked, 'what is the cause of human errors and violations in construction' using the construction site context. According to Reason [2], human error may include a deviation of some path, either from an intended course of action, from a planned of effort towards the desired goal, and from the appropriate behaviour at work. Violations are a type of mistakes, which happens when individuals fail to follow the instruction or decide to make their own rules when undertaking an activity [3]. In essence, violations are a deliberate act that often happens due to conscious decisions and actions of individuals in the workplace.

The outcomes of human errors are evident in social contexts, and their prevention could be addressed with a motivational process, and organisational remedies [3]. The notion by Lopez et al. [3] that errors manifest and evolve within a social context explains why

* Corresponding author: mfemuze@ cut.ac.za 
Reason [2] contends that regardless of a skill level, experience or training of people, mistakes and violations often find expressions in workplaces. In addition, deprived organisational deficiency in a workplace is a major source of human errors that contribute to accident causality [1].

To address the dilemma of this study, one needs to understand the causes of human error in construction. According to Dekker [4], there are two views of human errors: the old view and the new view. In the former, 'human error is a cause of accidents' and in the new view 'human error is a symptom of trouble deeper inside a system'. These two views might sound similar, but they are common in that when investigating human errors, you must understand why individuals did what they did (i.e. examining accident or system failure) and not to judge decision-making of the individuals [4].

\section{Literature Review}

Human failure is in two ways, the person approach and system approach [5]. The person approach focuses on either the mistake, error, slips or lapse of individuals by blaming them for forgetfulness, inattention, and moral weakness. The system approach focuses on the condition under which individuals work and tries to build defences to avert the error and mitigate their effects [5]. Human error is described as a systematically connected frame of individual tools, for example, tasks and operating environment [6]. Progress on the safety of individuals at the job site comes from understanding and influencing system connections [7]. One significant consequence of human errors is the manifestation of accidents.

\subsection{Accidents and latent failures}

An accident is an unforeseen event that results in a disagreeable consequence, which is linked to human activities instead of natural phenomena [1]. They are the central issue of safety management in any manual and mechanical industry, and the construction industry is no exception [8]. The reason is that the construction industry accounts for a high number of occupational fatal and nonfatal injuries because of accidents [9]. According to Albert et al. [9], collisions may be influenced by the underlying conditions from errors and violations. To address human mistakes and abuses, Reason et al. [10] used a model for illustrating the interventional mechanisms. The 'Swiss Cheese' accident causation model designed by Reason with the purpose to prevent accidents by defences that disallow risks and hazards that can cause loss (see Figure 1). The 'Swiss Cheese' model is divided into two groups namely, hard defence group that are automatic alarming system and the soft defence group which are depended upon the personnel and procedures of required performance [11].

As indicated in Figure 1, each slice of cheese has holes in it, and the gaps between the cheeses are in continues motion, moving from culture and leadership (staff shortage) to clinical support (poor team communication). The holes in the cheese slices represent individual weaknesses in individual parts of the system and are continually varying in size and position in all slices. The gaps arise from unsafe acts and latent failure, permitting "a trajectory of accident opportunity", so that a hazard passes through holes in all of the defences, leading to an accident [10]. Therefore, the latent failure shown in the holes in the cheese slices refer to events, activities, or conditions that deviate from expectations, and cause adverse consequences of organisational significance [12]. 


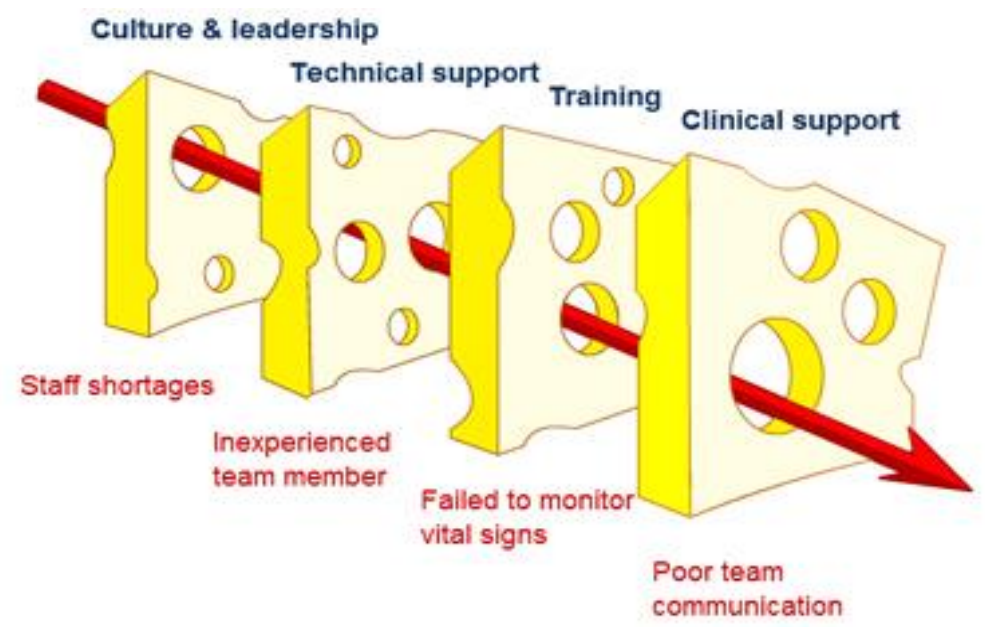

Fig. 1. Reason's 'Swiss Cheese' Model (Source: [10])

Therefore, latent failures could be deduced from an accident caused by the decisions and actions of construction professionals not directly involved in the frontline of physical site work. The failures are typically found in the safety system of a project [10]. Mostly, latent failings happen when there is a lack of supervision or inadequate understanding of the standard work procedure. Practitioners and researchers have argued that the causes of project failure could be traced to latent failure [8]. Latent failures are found in the work system as they merge with natural human tendencies and results in human error and violation of the event [13].

According to Salmon et al. [6], latent failures are inevitable human errors and are always present on construction sites. Most of the time, the latent failures are unforeseen, and they tend to be hidden and trigger at a later stage of the project causing accidents which either result in injuries or fatalities [14]. According to Ellison ([13] citing [2]), latent failure could be caused by limited resources supplied by management. There are numbers of factors causing latent failure in the industry include ineffective training, poor design of plant and equipment, inadequate supervision, ineffective communication, insufficient resources, and uncertainties in roles and responsibility [2]. These factors are technical enablers of latent failures in safety defence systems.

\section{Research Methodology}

This study aims to identify the causes of human errors and violations in construction. The focus of the reported research is poor safety practice on construction sites. To achieve this goal, an inductive research approach explained by Maxwell [15] was conducted. The reason for using an inductive research approach was to understand the problem through the lived experiences of practitioners. The primary data were synthesised with the reviewed literature in the subject areas. Notable sources include Reason [10], Reason [2] and Dekker [4]. The data of this study was collected using in-depth interview technique with a semistructured protocol as the instrument. The interviews were conducted on a construction site with several contractors. Some interviewees were provided with the protocol before the sessions through emails to ensure comprehension of the issues.

The interviewees were the member of the contractors working from different construction organisations specialising in the building and civil engineering projects. The primary data were collected from three major municipalities in the Eastern Cape Province of South Africa. 
In effect, contractors in three towns, which include Buffalo City Metropolitan Municipality, Ndlambe Local Municipality, and Nelson Mandela Bay Metropolitan Municipality, took part in the study. The selection of the contractors was purposive as explained by Maxwell [15] and the database of each municipality was used to identify contractors who have been appointed by the city to deliver their projects.

The interview sessions were between 15 to 30 minutes in duration. The interviewees who preferred questionnaire to be emailed to them took two to five days to respond to the emails sent to them. In total six (6) construction professionals were interviewed personally, and twenty (20) construction professionals responded to the questionnaire through email communication. In total 45 construction professionals were approached, and only six agreed to be interviewed, and thirty-nine (39) professionals were sent emails, and only twenty (20) responded to the email (see Table 1). The data were analysed using the human error and violations themes.

Table 1. The interview Sample.

\begin{tabular}{cccc}
\hline Participants & Male & Female & Percentage (\%) \\
\hline Construction managers & 4 & 2 & 23.1 \\
\hline Site engineers & 3 & 1 & 15.4 \\
\hline Foreman & 4 & 1 & 19.2 \\
\hline Quantity surveyors & 2 & 3 & 19.0 \\
\hline Artisans (skilled workers) & 6 & & 23.1 \\
\hline Total & $\mathbf{1 9}$ & $\mathbf{7}$ & $\mathbf{1 0 0 . 0}$
\end{tabular}

\section{Research Findings and Discussion}

\subsection{The impact of an organisational system that produces the human error}

Majority of the interviewees responded that they are using a different method to prevent human mistakes on sites and that the order they are using is determined by the scope and nature of the project. It is reported that a safety system is supposed to be designed according to the risk assessment plan, risk management plan, and quality control plan of projects. They further explained that project specifications are very significant as it often helps them to understand the details of the project design concerning hazardous activities. The responses of the interviewees appear to resonate with the importance of a safety system explained by Albert et al. [9] who opined that the fundamental goal of an effective safety system is to help organisations to eliminate or reduce safety risk before work begins on a construction site.

Majority of the respondents explained that they use toolbox talk to analyse the risks in an activity that they were about to undertake on a daily basis. For example, a foreman explained that they had designed a risk assessment checklist to highlight and identify actions that are dangerous and to help them to provide the right safety protective clothes for the task they are undertaking. An interviewee explained that he fails to understand why the construction industry is still experiencing a high rate of accidents despite the introduction of safety regulations to eliminate accidents and promote safety culture on sites.

It is reported that safety guidelines and safety warning boards are very significant to warn and remind the workers about hazardous and dangerous activity on sites. A construction manager and site engineer had a similar response about a safety signboard, which they argued should be designed using the home language of the area they are working on, instead of using the English language because local workers may not be fluent the English language. According to McAleenan and McAleenan [16], a site safety warning sign informs the workers 
that they are about to enter a 'dangerous site'. Furthermore, several authors regarding the famous saying that a language is a universal medium of communication corroborate a statement of a construction manager and a site engineer about the importance of language.

\subsection{Accidents on construction sites}

There are various factors causing accidents on construction sites, and such elements are linked to workers' decisions, actions and behaviours. Some interviewees argued that productivity is the leading cause of accidents. They explained that management always prioritises productivity more than the safety and well-being of workers. They contend that workers work under extreme working conditions and when they are tired, they tend to lose concentration to the extent that they would succumb to the temptation to take shortcuts, which end up causing accidents. This explanation is linked to a system approach as described by Reason [10].

Most of the interviewees responded that accidents had become a standard practice in their line of work. A health and safety manager states that to start and finish a project without experiencing a minor or major injury is a significant achievement, which deserves a celebration. Accidents in the construction industry is a substantial concern as explained by Albert et al. [9] who presented statistics that argued that in the United State of America (USA), construction workers are more likely to be killed or injured more than workers in other industries.

Furthermore, it is reported that failure by the employers and employees to follow and respect the organisational safety regulations, designed risk assessment tool and failure to set safety prevention measure will always contribute towards accidents on sites. A construction manager explained that most of the accidents they had experienced in their projects are the results of an error made either by the supervisor or foreman while workers are busy on an activity. This statement of a construction manager is surprising because often a member of the management always blames the workers for accidents, but in this case, both management and the workers are accused of error, which leads to accidents. The reason why human error is blamed on the employers and employees is explained by Dekker [4] with the so-called 'bad apple theory'. In this theory, Dekker [4] suggests that disastrous outcome are preventable if somebody had paid a bit more attention at the job site.

\subsection{The impact of the human violation on construction sites}

Majority of the interviewees responded that the leading causes of violation on a project site are workers. Furthermore, they explained that often workers fail to concentrate while undertaking an activity, especially if a supervisor is not present. It is clear from the start that violation on-site is blamed on the workers as Holden [7] states that the blame on people continues because it is both a fundamental psychological tendency as well as an industrystandard that remain strong in the human activity industry. In addition, it represents the person approach as explained by Reason [10] in the literature.

Furthermore, the interviewees expressed that workers are careless, and this is a significant problem, which organisation are struggling to handle. A foreman gave an example to support this statement that they had an incident where a worker had stepped on a nail while not wearing safety boots. Instead, the worker was wearing sneaker shoes because he complained that the safety boots make his feet to have a grump. This statement raises questions about organisational safety regulations. A foreman as a leader is not supposed to allow a worker to disrespect the organisational safety rule, and when an incident happened, he cannot blame the worker while he is the one who allowed this situation to occur under his watch. Furthermore, Reason [10] explained that often individuals may forget to be afraid 
at the workplace or that a nail might hurt his feet while wearing sneaker shoes, but the culture of a high-reliability organisation provides them with both the reminders and the tools to help them remember.

One of the interviewee, working on a road construction project responded that fatigue of the workers causes a human violation. He explained that the majority of their workers are the relatively young adults who do not rest because during the week they come to work, and on weekends, they go to parties. This explains why they often experience fatigue in construction work that is difficult and complex. Fatigue should therefore not pervade a worksite, which requires high energy and strength of the workers. He further explained that they have a breathalyser test to check the worker if they are trunk or not especially on the first Monday of the month. Some interviewees further explained that lack of payment causes a violation. They suggest that often the Government Department fail to pay them on time and because of this result, they fail to pay their skilled workers on time, and often they resign, and the organisations end up promoting unskilled workers to carry out activities that require skilled workers to do.

\section{Conclusions}

Worldwide, construction work is recognised as hazardous and accident-prone. While other sources exist, accidents in construction are linked to human errors and violations $[2,3]$. Furthermore, it is reported in the literature that infringements are a type of errors or mistake, which happens when workers fail to follow instructions. In effect, contractors should pay closer attention to site operations to identify and eliminate factors causing human errors. It was discovered that most of the interviewees are aware of the impact of human errors and violations. The interviewees cited ways in which human errors and violations could be reduced. The use of a safety signboard to warn the workers and visitors about hazardous activities serves as an intervention in this context. In conclusion, this study is recommending that training workshop should be introduced to individuals to teach them how to identify and eliminate human errors and violations in the construction

\section{Acknowledgement}

The first author has drawn on treatises produced by his students in 2017. The authors gratefully recognise the following students; Luyolo Sigudla, Lubabalo Mabentsela, Bonisile Xashe, Genevieve Coetzee, Amava Ndinisa, and Keletso Letosu.

\section{Reference}

1. E. Hollnagel, B arriers and A ccident Prevention. A shgate Publishing Limited, A Idershot, (2004)

2. J. Reason, Human Errors and M odels M anagement. Cambridge: TECNATOM (2011)

3. R. Lopez, E. Peter, D. Love, D.J. Edwards, Design error classification, causation in construction engineering, Journal of Performance of Constructed Facilities, 24(2) (2014)

4. S. Dekker, Field Guide to Understand 'Human Error', A shgate Publishing L td (2014)

5. J. Reason, Human error: models and management, BMJ 320: 768-770 (2000)

6. P.M. Salmon, M. Cornelissen, M.J. Trotter, Systems-based accident analysis methods: a comparison of A cciM ap, HFACS and STA M P, Safety Science 50(4): 206-220 (2012)

7. R.J. Holden, People or systems? To blame is human. The fix is to engineer, National Primary Care (PMC) 54(12) (2009) 
8. P. Promsorn, P. Soponsaul rat, C.A. A dulyamukosol, P. K aiyarit, T. Chinda, Identifying root causes of construction accidents: non-human error factors, Journal of communication and instrument 2(1): 4 (2015)

9. Albert, M.R. Hallowell, B.M. Kleiner, Emerging strategies for construction safety $\&$ health hazard recognition, Journal of Safety, Health \& Environmental Research 10(2) (2014)

10. J. Reason, The Human Contribution: Unsafe Acts, Accidents and Heroic Recoveries. N ew Y ork, USA : Routledge (2008)

11. S.S. Hosseinian, Z.J. Torghabeh, Major theories of construction accident causation models, International journal of advanced in engineering 4(2) (2012)

12. R. Ramanujam, P.S. Goodman, Latent errors and adverse organizational consequences: a conceptualization, Journal of Organizational Behavior 24: 815-836 (2003)

13. M. Ellison, Construction law made easy, CmCK omodo (2014)

14. S. K um, B. Sahin, A root analysis for A rctic M arine A ccidents from 1993 to 2013, Safety Science 74 (2015)

15. J.A. Maxwell, Qualitative Research Design: An Interactive Approach. (3rd ed.), California, USA, Sage Publications (2013)

16. M CA leenan, P. M cA leenan, Critical Theory: Understanding the Impact L anguages has on Workers Safety and Health, Proceedings of Institution of Civil Engineers: Management, Procurement and Law, 170(M P2): 52-58 (2017) 